\title{
Sporamin Intervention Altered the Gut Microbiome and Tumor Tissue Transcriptome in Mice Intraperitoneally Xenografted With the Human Colorectal Carcinoma LoVo Cells
}

Chun Yang

Capital Medical University

Bo-Wen Chen

Capital Medical University

Kai-Wen Zhang

Capital Medical University

Si-Jia Chen

Aerospace central Hospital

Xiao-Peng Zhang

Lai Zhou People's hospital

Jing-Jie Zhang

China National Children's Center

Ge Guo

China National Center for Food Safety Risk Assessment

Peng-Gao Li ( $\square$ penggao@ccmu.edu.cn )

Capital Medical University

Research

Keywords: Sporamin, Colorectal cancer, Microbiome, Transcriptome, 16S rRNA sequencing, RNA sequencing

Posted Date: September 28th, 2021

DOl: https://doi.org/10.21203/rs.3.rs-923879/v1

License: (1) This work is licensed under a Creative Commons Attribution 4.0 International License. Read Full License 


\section{Abstract}

\section{Background}

Accumulating evidence indicates that sporamin, the main storage protein in the sweet potato (Ipomoea batatas), can suppress the development of colorectal cancer (CRC), but the changes in the gut microbiome after sporamin intervention and its relationships with the pathogenesis of $\mathrm{CRC}$ have not been investigated.

\section{Methods}

Twelve male athymic BALB/c nude mice were randomly divided into four groups, CG1, CG2, TCG, and TTG. Mice in TCG and TTG were intraperitoneally transplanted with the LoVo cancer cells before the interventions started. CG2 and TTG were intragastrically infused with sporamin $(0.5 \mathrm{~g} / \mathrm{kg} \mathrm{BW} / \mathrm{day})$ for four weeks while CG1 and TCG were infused with the same volume of water during the experiment. Fecal samples were collected after the interventions and then examined for the changes in the microbiota using the $16 \mathrm{~S}$ ribosomal RNA (rRNA) sequencing technology. The functional capabilities of the gut microbiota were predicted with the PICRUSt pipeline. Transcriptomic profiling of the tumor tissues was carried out for tumor-bearing mice with the RNA-sequencing (RNA-seq) technology and the resultant differentially expressed genes (DEGs) were then analyzed in terms of gene ontology (GO), protein-protein interaction (PPI), transcription factors (TF) prediction, and biological pathway annotations.

Results

Sporamin significantly reduced the tumor burden of tumor-bearing mice and brought beneficial changes to the gut microbiome in both kinds of mice. It significantly increased the proportions of Barnesiella and Lactobacillus but reduced that of Bacteroides in tumor-bearing mice. The phenylalanine metabolism pathway, the glyoxylate, dicarboxylate metabolism, the bacterial secretion system, the glycan biosynthesis and metabolism, and the biosynthesis of stilbenoid, diarylheptanoid, and gingerol were favorably modulated by sporamin intervention. Sporamin mainly modulate the expression of the genes involved in the protein processing in the endoplasmic reticulum, the glycosylphosphatidylinositol (GPI)anchor biosynthesis pathway, and the mineral absorption pathway.

\section{Conclusion}

Sporamin could favorably alter the gut microbiome and its metabolome, improving the gut microenvironment and the viability of the gut microbiota and increasing the detoxification and bioactive substance production activities in the large intestine, by which the host's metabolome may be altered and in turn exerts a suppressing effect on the protein synthesis and growth of tumor tissues.

\section{Background}


Colorectal cancer (CRC) is one of the most prevalent gastrointestinal cancers worldwide. It is a major contributor to worldwide cancer mortality and morbidity and, in 2018, over 1.8 million new cases and 881,000 deaths were reported [1]. Its etiology is very complex that involves multiply risk factors, such as genetic susceptibility, environmental toxins, lifestyle and dietary factors, and their complex interactions [2]. However, to date, the interactions between these factors have been very difficult to study and explain. On the other hand, the effective therapeutic strategies for CRC are also hampered by many limitations such as drug resistance and low long-term survival. Therefore, early prevention still is the best way to reduce the cancer burden.

In this regard, the crucial roles played by the dietary factors in the prevention and treatment of CRC have been increasingly recognized [3]. They can influence the onset and development of CRC through multiple mechanisms such as regulating the gut microbiota dysbiosis, influencing the integrity and function of the intestinal mucosal barriers, modifying the intestinal immune responses that may lead to inflammatory bowel diseases and CRC $[4,5]$. In this process, the gut microbiome may be one of the key mediators that can modify the process both directly through contact with the intestinal mucosa and indirectly through fermenting the intestinal contents and changing the secondary metabolites produced [5-7]. In addition, dietary factors and gut microbiota also play an important role in modulating the host's response to chemotherapeutics and other oral treatments by influencing their bioavailability, toxicity, and efficacy [8, 9]. Consequently, using probiotics and/or prebiotics to intentionally modulate the gut microbiota and favorably influence the host's immune response and prevent the progression of CRC has been proposed as a potential therapeutic strategy [10]. What's more, more and more foods and food-derived bioactive components that are efficacious in reversing gut microbiota dysbiosis and preventing the development of CRC have been found [11-13]. This may open up a new avenue for the chemoprevention of CRC.

Sporamin is the major storage protein of the sweet potato (Ipomoea batatas) roots [14]. It can suppress the growth of a variety of cancer cells in vitro and in vivo [15-19]. However, to date, the impact of sporamin consumption on the host gut microbiome has not been explored, particularly in the context of CRC. Therefore, in the present study, the changes in the gut microbiome following oral administration of sporamin for four weeks were investigated in both healthy and tumor-bearing mice by using the bacterial 16S rRNA sequencing technology. The formation of tumors from the intraperitoneally (i.p.) transplanted human colorectal cancer LoVo cells was evaluated and the transcriptomic profiles of the tumor tissues after sporamin intervention were investigated by using the high throughput RNA sequencing (RNA-seq) technology. The potential molecular targets and mechanisms of action of sporamin were evaluated according to the changes in the gut microbiome and the tumor tissue transcriptome as well as their possible interactions.

\section{Methods}

\section{Materials}


Sporamin was extracted from fresh sweet potatoes as previously reported [20]. The human colorectal cancer LoVo cell line (Tumor Cell Bank of the Chinese Academy of Medical Sciences, Beijing, China) were cultured in Dulbecco's Modified Eagle's Medium (DMEM) (Thermo Fisher Scientific, Shanghai, China) supplemented with $10 \%$ fetal bovine serum (Thermo Fisher Scientific, Shanghai, China) and $1 \%$ penicillin-streptomycin at $37^{\circ} \mathrm{C}$ in a humidified incubator with $5 \% \mathrm{CO}_{2}$. Male BALB/c athymic nude mice (Vital River Laboratory Animal Technology Co. Ltd., Beijing, China) aged 6 weeks were housed in an access-restricted room with a 12-h/12-h light/dark cycle, controlled temperature $\left(22^{\circ} \mathrm{C}\right.$, daily temperature fluctuations $\leq 3^{\circ} \mathrm{C}$ ), relative humidity of $40 \%-70 \%$, an airflow velocity $\leq 0.18 \mathrm{~m} / \mathrm{s}$, and a room air pressure gradient of 20-50 $\mathrm{Pa}$ under specific pathogen-free conditions.

\section{Experimental design}

The experiment protocol was approved by the institutional ethics committee at Capital Medical University (license number: AEEI-2016-018). After a one-week acclimation period, twelve BALB/c athymic nude mice were randomly divided into four groups ( $n=3$ per group). $\otimes$ CG1: healthy mice treated with vehicle (control group); $\triangle \mathrm{CG} 2$ : healthy mice treated with sporamin; $\triangle \mathrm{TCG}$ : tumor-bearing mice treated with vehicle; $\triangle \mathrm{TTG}$ : tumor-bearing mice treated with sporamin. Mice in the CG1 group were intragastrically infused with 0.5 $\mathrm{mL}$ of distilled water (vehicle) per day for four weeks (28 days). Mice in the CG2 group were intragastrically infused with $0.5 \mathrm{~mL}$ of sporamin at $0.5 \mathrm{~g} / \mathrm{kg} \mathrm{BW}$ per day in distilled water for four weeks. Mice in the TCG group were i.p. transplanted with $2.5 \times 10^{7}$ LoVo cells in $0.2 \mathrm{~mL}$ of PBS and then intragastrically infused with $0.5 \mathrm{~mL}$ of distilled water (vehicle) daily for four weeks. Mice in the TTG group were i.p. xenografted with $2.5 \times 10^{7}$ LoVo cells in $0.2 \mathrm{~mL}$ of PBS and then intragastrically infused with 0.5 $\mathrm{mL}$ of sporamin at $0.5 \mathrm{~g} / \mathrm{kg}$ BW per day in distilled water for four weeks. All animals had free access to feed and drinking water throughout the experiment. Their body weights (BW) were recorded every 4 days during the experiment. At the end of the experiment, all mice were anesthetized with $10 \%$ chloral hydrate $(0.1 \mathrm{~mL} / 10 \mathrm{~g} \mathrm{BW})$ and sacrificed by cervical dislocation.

\section{Sample collection}

Fresh fecal samples were collected from the mice under sterile conditions the day before sacrificing and were stored at $-80^{\circ} \mathrm{C}$ until analysis. An autopsy was performed and tumors formed in the abdominal cavity were carefully examined, counted, measured for weight and size, collected and washed with RNAlater ${ }^{T M}$ Stabilization Solution (Thermo Fisher Scientific, Shanghai, China), and stored at $-80^{\circ} \mathrm{C}$ until analysis.

\section{Bacterial genomic DNA extraction, sequencing, and bioinformatic analysis}

Bacterial genomic DNA was extracted from 0.18 to $0.22 \mathrm{~g}$ of the fecal samples using the E.Z.N.A ${ }^{\circledR}$ MagBind Soil DNA Kit (Omega Bio-Tek, GA, USA) according to the manufacturer's instructions. The V3-V4 hypervariable region of the bacterial $16 \mathrm{~S}$ ribosomal RNA gene was amplified by polymerase chain reaction (PCR) using primers 341F 5囚-CCCTACACGACGCTCTTCCGATCTG-barcodeCCTACGGGNGGCWGCAG-3囚 and 805R 5囚- 
GACTGGAGTTCCTTGGCACCCGAGAATTCCAGACTACHVGGGTATCTAATCC-3\ under the following condition: $94^{\circ} \mathrm{C}$ for $3 \mathrm{~min}$, followed by five cycles at $94^{\circ} \mathrm{C}$ for $30 \mathrm{~s}, 45^{\circ} \mathrm{C}$ for $20 \mathrm{~s}$ and $65^{\circ} \mathrm{C}$ for $30 \mathrm{~s}$; this was followed by another 20 cycles at $94^{\circ} \mathrm{C}$ for $20 \mathrm{~s}, 55^{\circ} \mathrm{C}$ for $20 \mathrm{~s}$ and $72^{\circ} \mathrm{C}$ for $30 \mathrm{~s}$, with a final extension at $72^{\circ} \mathrm{C}$ for $10 \mathrm{~min}$. An 8-bp barcode sequence, unique to each sample, was attached to the primers for multiplexing. PCR was performed in triplicate in $50 \mu \mathrm{L}$ reaction mixtures containing $5 \mu \mathrm{L}$ of $10 \times \mathrm{PCR}$ buffer, $0.5 \mu \mathrm{L}$ of $10 \mathrm{mM}$ dNTPs, $10 \mathrm{ng}$ of genomic DNA, $0.5 \mu \mathrm{L}$ of each primer $(50 \mu \mathrm{M})$, and $0.5 \mu \mathrm{L}$ of Plantium Taq polymerase $(5 \mathrm{U} / \mu \mathrm{L})$. The PCR products were separated by $1.2 \%$ agarose gel electrophoresis, and bands of the desired size (> $600 \mathrm{bp}$ ) were purified using the SanPrep DNA Gel Extraction Kit (Sangon Biotech, Shanghai, China) according to the manufacturer's instructions.

Then, the bacterial genomic DNA samples were sent to Sangon Biotech Co. Ltd. (Shanghai, China) for 16S rRNA sequencing, where the DNA concentration of each PCR product was determined using a Qubit2.0 DNA fluorometer (Thermo Fisher Scientific, Shanghai, China). Equimolar purified products were pooled, and paired-end sequenced $(2 \times 300)$ on an Illumina MiSeq PE300 platform (Illumina, San Diego, CA, USA) according to the standard protocol at Sangon Biotech.

After sequencing, demultiplexing and quality-filtering of the raw fastq files were performed using QIIME (version 1.8.0) [21]. UCHIME [22] was applied to identify and remove chimeric sequences.

UPARSE [23] was used to cluster operational taxonomic units (OTUs) with a 97\% similarity cutoff level according to Greengenes (version 13.8). RDP Classifier [24] was used to assign sequences generated from samples to the corresponding taxonomy with a confidence threshold of 70\% [25]. The alphadiversity indices, including Chao1, ACE, Shannon, and Simpson, were calculated to summarize the richness, evenness, and distributions of the gut bacterial community. The principal coordinates analysis (PCoA) based on the weighted UniFrac distance matrix and the hierarchically clustered heat maps were constructed in $\mathrm{R}$ (version 3.2) to assess the beta-diversity of the gut microbiome across groups. Phylogenetic Investigation of Communities by Reconstruction of Unobserved States (PICRUSt) [26] was used to predict the functional potential of the gut microbiome based on the OTU table, where information relating to gene annotations was obtained from the Kyoto Encyclopedia of Genes and Genomes (KEGG) database. The abundance of each functional category was calculated according to OTU abundance and STAMP (version 2.1.3) [27] was used to analyze differential metabolic pathways across groups.

\section{Transcriptome profiling of the tumor tissues}

Tumor tissue samples from the TCG and TTG group were sent to Beijing Genomics Institute (BGI, Wuhan, China) for RNA extraction and sequencing (RNA-seq) according to the following procedures: (i) $1 \mu \mathrm{g}$ of the total tissue RNA was extracted using the TRIzol ${ }^{\circledR}$ reagent and treated with DNasel at $37^{\circ} \mathrm{C}$ for 20 min to digest DNA. Oligo dT magnetic beads were used to select mRNA with polyA tail; (ii) the purified mRNA were fragmented and reverse transcribed to double-strand cDNA (dscDNA) by using the N6 random primer (Sequence: 5' d(N6) 3' [N=A, C, G, T]); (iii) the ds cDNAs were treated by a traditional process, including end repairing with phosphate at the 5 ' end and stickiness ' $A$ ' at the 3 ' end, ligation, and adaptor 
with stickiness ' $T$ ' at the 3 ' end; (iv) two specific primers were used to amplify the ligation product; (v) the double-stranded PCR products were heat-denatured to single-strand and circularized by splint oligo and DNA ligase; (vi) sequencing was performed on the BGISEQ-500 platform with the prepared library. (vii) bioinformatics: low-quality reads, reads with adaptors, and reads with unknown bases were filtered to get clean reads and were stored in FASTQ format [28]. The clean reads were aligned to reference genome sequences via Bowtie2 [29]. Gene and isoform expression levels were quantified with RSEM [30]. Differentially expressed genes (DEGs) were detected with the DEseq2 package in $\mathrm{R}$ [31]. The criteria for selecting DEGs were fold-change $\geq 2.00$ and adjusted $P$-value $<0.05$. Hierarchical clustering analysis for DEGs was performed by the pheatmap package in R. Gene ontology (GO) and pathway enrichment analysis based on the KEGG database were performed online. Transcription factor (TF) prediction was performed by using the DIAMOND [32] combined with the information recorded in the human TF database (Human TFDB). A protein-protein interaction (PPI) network was plotted using the STRING database [33] and the Cytoscape network analysis platform [34].

\section{Real-time quantitative reverse transcription PCR (RT-qPCR)}

To validate the DEGs obtained from RNA-seq, the validation experiments were carried out by using RTqPCR. The total RNA of tumor tissues was extracted with the TRIzol ${ }^{\circledR}$ reagent (Thermo Fisher Scientific, Shanghai, China). The quality and yield of RNA were determined with a NanoDrop ${ }^{\circledR}$ ND-1000 ultraviolet spectrophotometer (Thermo Fisher Scientific, Shanghai, China). Complementary DNA was reversetranscribed using the RevertAid First Strand cDNA Synthesis kit (Thermo Fisher Scientific, Shanghai, China). RT-qPCR was conducted with a Bio-Rad real-time PCR detection system (Bio-Rad, MA, USA) using KAPA SYBR ${ }^{\circledR}$ FAST qPCR Master Mix Kit (KAPA Biosystems, MA, USA) according to the manufacturer's instructions. The specific primers for the selected genes are provided in Supplementary Table S1. All reactions were performed in triplicate under the following procedures: $95^{\circ} \mathrm{C}$ for $10 \mathrm{~min}$, followed by 37 cycles of $95^{\circ} \mathrm{C}$ for $15 \mathrm{~s}, 60^{\circ} \mathrm{C}$ for $30 \mathrm{~s}$ and $72^{\circ} \mathrm{C}$ for $30 \mathrm{~s}$, and a final extension at $72^{\circ} \mathrm{C}$ for $10 \mathrm{~min}$. The Ct values of the genes were used to calculate the degree of gene expression using the $2^{-\triangle \Delta C T}$ method normalized to that of the $\beta$-actin gene [35].

\section{Statistical Analysis}

Data are expressed as mean \pm SEM (standard error of the mean). Differences among groups were examined by one-way analysis of variance (ANOVA) using SPSS Statistics version 18.0 (IBM, New York, USA). The Turkey-Kramer Post-hoc test was used when the homogeneity of variance was equal between groups. The Game-Howell test was used when the variance was unequal. $P<0.05$ was considered statistically significant.

\section{Results}

\section{Sporamin alleviated the tumor burden of mice without any observable adverse effects}


During the experiment, the healthy mice in the CG2 group showed no obvious side effects compared with the control mice in the CG1 group, indicating that oral consumption of sporamin is safe for healthy mice. For tumor-bearing mice in the TCG and TTG group, all of them developed tumors in their abdominal cavities after about eight days of the i.p. transplantation of the LoVo cells, which became touchable and bulged gradually, indicating that the transplantation was successful. During the experiment period, tumorbearing mice (the TCG group in particular) were listless, inactive, and slightly thinner than their counterparts in the sporamin intervention group (TTG) who were more physically active and mentally poise. Throughout the experiment period, the BW of the mice were comparable across the four groups, indicating that consumption of sporamin for four weeks does not significantly influence the growth of the mice $(P>0.05$, Figure $1 \mathrm{~A})$. Autopsy of tumor-bearing mice revealed that a great number of tumors were grown in the abdominal cavity of the mice in the TCG group, which were greatly relieved by sporamin intervention in the TTG group (Figure 1B, C).

\section{Sporamin intervention favorably changed the gut microbiome and its metabolome}

The 16S rRNA sequencing of the fecal samples generated 834,275 usable reads from twelve samples with a mean length of $421.41 \pm 7.93 \mathrm{bp}$ per read. The sequencing coverages for all samples were above $99 \%$. There were $256.33 \pm 32.32,244.67 \pm 35.02,235.33 \pm 23.80$, and $269.00 \pm 22.91$ OTUs identified for the CG1, CG2, TCG, and TTG group, respectively. Flattening of the rarefaction curves based on species richness and Shannon indices (Supplementary Figure S1) indicates that our data volume and sequencing depth were sufficient. There were no significant differences in the alpha-diversity indices, including the ACE, Chao1, Shannon, and Simpson indices, across the four groups $(P>0.05)$ (Supplementary Table S2).

To explore the dissimilarities of bacterial communities across groups (beta-diversity), the PCoA and hierarchically clustered heat maps were constructed. The three-dimensional PCoA plot (Figure 2A) shows that $45.4 \%, 23.8 \%$, and $12.0 \%$ of the dissimilarities between the samples could be explained by PCoA1, PCoA2, and PCoA3, respectively. The two-dimensional PCoA plot (Figure 2B) clearly shows that the healthy mice (CG1 and CG2) mainly locate in the second quadrant, indicating that consumption of sporamin did not significantly alter the structure of the gut microbiome in the healthy mice. This is consistent with its impacts on the BW of mice. Tumor-bearing mice (TCG) tend to aggregate to the fourth quadrant, indicating that the growth of the xenografted tumors had significantly altered the structure of the gut microbiome, which were partly pulled back to the first and the second quadrant after sporamin intervention (TTG), suggesting that sporamin intervention could normalize the abnormal structure of the gut microbiome in tumor-bearing mice. To look at the changes more closely, hierarchically clustered heat maps (Figure 2C) illustrating the differential composition of the fecal bacterial communities at the phylum and genus level were constructed, which show that mice who had received the sporamin intervention (CG2 and TTG) were clustered into a subgroup while those who had not received the intervention (CG1 and TCG) were clustered into the other subgroup, indicating that the consumption of sporamin had brought a significant impact on the taxonomic composition of the microbiome of both healthy and tumor-bearing mice. 
Therefore, we further compared the differences in the relative abundances of the bacterial taxonomies after sporamin treatment. Figure $3 \mathrm{~A}$ shows that there were eight bacterial phyla and an archaea phylum, Euryarchaeota, identified in all fecal samples, among which, Bacteroidetes and Firmicutes were the two predominant phyla in all animals. After tumor transplantation, the proportion of Bacteroidetes was further increased while those of other phyla were correspondingly reduced. In comparison, sporamin intervention decreased the proportion of Bacteroidetes and increased that of Firmicutes in tumor-bearing mice, and subsequently increased the Firmicutes/Bacteroidetes ratio to a more balanced level. The details of the relative abundances of all phyla are provided in Supplementary Table S3. The distribution histogram of the gut microbiome at the genus level (Figure 3B) shows that 48 bacterial genera were identified in all samples. Sporamin intervention increased the proportions of Muribaculaceae and Oscillibacter in healthy mice. Notably, the proportion of Bacteroides increased greatly after tumor transplantation, which was significantly inhibited by sporamin intervention. In addition, the proportions of Barnesiella and Lactobacillus were significantly increased by sporamin intervention in tumor-bearing mice. The details of the changes in the bacterial communities at the genus level are provided in Supplementary Table S4.

To explore the functional relevance of the changes in the bacterial compositions brought by sporamin intervention, the 16S rRNA data were submitted to the PICRUSt for functional prediction. Figure 4A shows that sporamin did not significantly alter the metabolic pathways that were inferred from the phylogenetic composition of the gut microbiome in the healthy mice (CG1 \& CG2), but transplantation of the LoVo tumor cells led to a significant increase in the functional abundance of the proximal tubule bicarbonate

reclamation pathway (from $0.02 \%$ to $0.03 \%, P=0.03$, Figure $4 \mathrm{~B}$ ) compared with control mice, implying that the acid-base balance and the kidney function of tumor-bearing mice might have been impaired by the microbiome-related factors, which disappeared after sporamin intervention (Figure 4C). In addition, in tumor-bearing mice, sporamin intervention significantly increased the relative proportions of the bacterial secretion system pathway $(P=0.04)$, the glycan biosynthesis and metabolism pathway $(P=0.04)$, and the stilbenoid, diarylheptanoid, and gingerol, biosynthesis pathway $(P=0.04)$ while significantly reduced the proportions of the phenylalanine metabolism pathway $(P=0.02)$ and the glyoxylate and dicarboxylate metabolism pathway $(P=0.03)$, implying that sporamin intervention may exert a significant impact on the development of tumor through modulating the metabolome of the gut microbiome and then alter the pathophysiology of the host such as its intestinal barrier function, immune responses, and other cancerrelated metabolisms, etc.

\section{Sporamin changed the transcriptome of the tumor tissues}

To explore the possible molecular targets of the sporamin intervention in tumor-bearing mice, the gene expression profile of the xenografted tumor tissues after sporamin intervention was investigated using RNA-seq technology. As a result, the sequencing generated $24065679 \pm 21370$ raw sequencing reads and $24066787 \pm 21381$ clean reads after filtering out low-quality reads. The numbers of the reads and their quality metrics for each sample are provided in Supplementary Table S5. The average mapping ratio with the reference genome was $85.25 \%$; the average mapping ratio with the reference genes was $69.10 \%$ and a 
total of 21,655 genes were detected. RSEM-quantified gene expression levels and the numbers of identified expressed genes in each sample are shown in Supplementary Figure S2.

Fourteen DEGs were identified in tumor-bearing mice after sporamin intervention (Figure. 5A). Eleven of them were upregulated (BCL11A, HSPH1, CHORDC1, SLC26A9, NEMF, HSPA4L, NIPBL, CHD1, ZNF638, ZBED6, HSP90AA1) while three of them were down-regulated (PRH1-PRR4, EDN2, PIGL) after sporamin intervention. The comparisons of the expression patterns of the DEGs in the two groups are shown in Figure. 5B. Except for CHORDC1, ZNF638, and PRH1-PRR4, the expressions of the remaining DEGs were confirmed by an independent RT-qPCR experiment (Figure. $5 C$ ).

To elucidate the functional interplays between the proteins encoded by these DEGs, a protein-protein interaction (PPI) network was constructed using the homology with the known PPIs reported in the STRING database (Figure 5D), which revealed a PPI network composed of seven proteins (i.e. HSP90AA1, HSPH1, HSPA4L, NEMF, CHORDC1, CHD1, and NIPBL) that were all upregulated after sporamin intervention.

The functional relevance of these DEGs was also analyzed by the gene ontology (GO) analysis in terms of the three classifications: biological process (BP), cellular component (CC), and molecular function (MF). Figure 6A shows that, in the CC category, eleven DEGs were annotated to cell and cell party; ten were annotated to organelle. In the MF category, eleven DEGs were annotated to binding; three were annotated to catalytic activity. In the BP category, twelve DEGs were annotated to positive regulation of the cellular process; eleven were annotated to positive regulation of the metabolic process.

Then, the critical biological pathways associated with the DEGs were investigated based on the KEGG database (Figure 6B) and found that three pathways (i.e. protein processing in endoplasmic reticulum, glycosylphosphatidylinositol (GPI)-anchor biosynthesis pathway, and mineral absorption pathway, respectively) were significantly enriched for the DEGs found between the TCG and TTG group. The KEGG-DEG relationship network analysis (Figure 6C) revealed the DEGs that are responsible for the promotion of the protein processing in the endoplasmic reticulum (i.e. HSPH1, HSP90AA1, HSPA4L), the increase in the mineral absorption pathway (i.e. SLC26A9), and the reduction in the GPIanchor biosynthesis pathway (i.e. PIGL) after sporamin intervention.

Then, in the TF prediction analysis, four TFs were found upregulated after sporamin intervention in tumorbearing mice, i.e. NEMF, HSP90AA1, BCL11A, and ZBED6. Their activities are closely related with three zinc finger domains (i.e. zf-C2H2, zf-C2HC, and zf-BED, Figure 6D), implying that sporamin may increase their transcriptional activities by increasing the absorption and bioavailability of zinc ions in their activity center.

\section{Discussion}

In the current study, we demonstrated for the first time that oral consumption of sporamin for four weeks could alter the composition and function of the gut microbiome and the transcriptome of the tumor 
tissues in tumor-bearing mice that were i.p. xenografted with human colorectal cancer LoVo cells. Sporamin mainly exerts its role on the gut microbiome by increasing the relative abundances of Barnesiella and Lactobacillus and reducing that of Bacteroides in tumor-bearing mice. As a result, the metabolome of the gut microbiome was changed, which possibly mediated part of the inhibitory effect of the sporamin intervention on the growth of the tumor. Transcriptomic analysis of the tumor tissues revealed that sporamin mainly upregulated the genes involved in the protein processing in the endoplasmic reticulum and the mineral absorption pathway, and reduced the GPI-anchor biosynthesis pathway, thereby exerting a suppressive role in the growth of the xenografted tumor.

Sporamin has been previously shown to play an anti-cancer role in a diversity of cancers in vitro and in vivo [15-19]. However, its mechanisms of action in the context of different cancers have not been completely understood. Because it has been proved to be a potent trypsin inhibitor that can inhibit the activity of the main digestive enzymes [20], it is reasonable to hypothesize that it can survive gastrointestinal digestion and reach the large intestine and interact with the microbiome there to exert an effect on the host. This is possibly an important route by which sporamin alters the metabolism, immunity, and pathophysiology of the host and suppresses the growth of cancers in the body. Our findings that consumption of sporamin for four weeks significantly altered the gut microbiome and its metabolome in tumor-bearing mice confirmed this hypothesis, suggesting that favorably improving the gut microbiome and its metabolome may at least partly mediate its anti-cancer effect on the host.

Regarding the etiology and pathology of CRC, it has been recognized that genetic susceptibilities, dietary patterns, and environmental toxins in combination are responsible for the onset and development of the disease. There are a lot of potentially harmful factors in the environment where the intestinal epithelial cells live, among which the intestinal microbiota constitute one of the most pertinent and persistent components of the intestinal microenvironment [36,37] and are closely associated with the pathogenesis of malignancies in the intestinal tract [38]. Some intestinal bacteria and their metabolites can stimulate inflammation and cause DNA damage and contributing to the occurrence and development of CRC [39]. On the other hand, some other intestinal bacteria, such as short-chain fatty acid (SCFA)producing bacteria, have been shown to favorably influence the health of the gut epithelia and prevent their malignant transformation. In line with this, improving the composition and function of the gut microbiota via prebiotics or probiotics or antibiotics etc. has also been proposed as a method to prevent cancer and is successful in several studies $[5,7,11]$. Moreover, the scope of the agents that can be used to modulate the gut microbiome is expanding. More and more natural products that are not mainly composed of dietary fibers are potent gut microbiota modulators and may exert beneficial roles in a variety of diseases $[13,40,41]$.

In the present study, the proportion of Bacteroidetes increased significantly after tumor transplantation, which was partially reduced by sporamin intervention, resulting in a more balanced Firmicutes/Bacteroidetes ratio. At the genus level, sporamin intervention significantly increased the relative abundance of Barnesiella and Lactobacillus and reduced that of Bacteroides in tumor-bearing mice. Barnesiella is decreased in CRC patients [42] and plays anti-inflammatory, anti-cancer roles in a 
colitis-associated CRC model through increasing the production and secretion of acetate, butyrate, and propionate in the gut [43]. As to Lactobacillus, it is a widely accepted probiotic that demonstrates beneficial roles in preventing CRC $[44,45]$.

Prediction of the functional potentials of the gut microbiome by PICRUSt indicated that the proximal tubule bicarbonate reclamation function was elevated in tumor-bearing mice, suggesting that they may be under the threat of acidosis and need to increase their bicarbonate reclamation capacity to maintain the acid-base balance [46]. Sporamin intervention abolished this elevation and produced five other favorable changes in the metabolic pathways. First, sporamin reduced the activity of the phenylalanine metabolism pathway. A study involving a large cohort of multi-omics data indicated that the activity of the phenylalanine metabolism pathway led to an increase in intramucosal carcinomas in the colon [47]. A product of phenylalanine degradation, benzene-oxide, is reactive towards proteins and DNA and is potentially carcinogenic [48]. Second, sporamin intervention reduced the activity of the glyoxylate and dicarboxylate metabolism pathway that is enriched in colorectal tumor tissues [49]. Chronic exposure of breast epithelial cells to the product of glyoxylate oxidation, oxalate, has been found to promote the transformation of normal cells to tumor cells by inducing the expression of the c-fos proto-oncogene and the proliferation of cancer cells [50]. A reduction in this pathway might increase the abundance of Lactobacillus [51] and Enterococcus [52] which was also observed in the present study and is beneficial for CRC. Third, sporamin intervention increased the activity of the glycan biosynthesis and metabolism pathway, indicating that sporamin might promote the overall survival and growth of gut bacteria because the synthesis of glycans in the cell wall is vital for bacteria [53]. Future studies should aim to quantify the total number of bacteria in the gut after sporamin intervention. Fourth, sporamin intervention increased the activity of the bacterial secretion system that might also be a reflection of an improved survival environment for the gut microbiome after sporamin intervention because the secretory function is an essential reflection of the viability of the gut microbiota. Fifth, sporamin intervention increased the stilbenoid, diarylheptanoid, and gingerol, biosynthesis pathways that might be also attributed to an overall increase in bacterial viability so that they can ferment the intestinal contents and produce more plant-derived bioactive compounds. Collectively, changes in these pathways after sporamin intervention suggest that the gut microbiome and its metabolome may at least partly mediated the beneficial role of sporamin intervention in tumor-bearing mice. Sporamin intervention may alter the pathophysiology of the host such as its intestinal barrier function, immune responses, and other cancer-related metabolisms via modulating the gut microbiome.

Examination of the transcriptome of the tumor tissues after sporamin intervention revealed that it mainly targets genes whose protein products participate in the protein processing in the endoplasmic reticulum (i.e. HSP90AA1, HSPH1, and HSPA4L), the GPI-anchor biosynthesis pathway (i.e. PIGL), and the mineral absorption pathway (i.e. SLC26A9). TF predictions identified four TFs (i.e. ZBED6, BCL11A, HSP90AA1, and NEMF), the activity of which are related to three conserved DNA binding zinc finger domains (i.e. zf-C2H2, zf-C2HC, and zf-BED). Among them, ZBED6 (zinc finger, BED-type containing 6) modulates gene expression primarily by repressing transcription. The gene targets of ZBED6, including the Wnt, Hippo, TGF- $\beta$, EGF receptor, or PI3K pathway components, are all involved in CRC 
development [54]. BCL11A (B-cell lymphoma/leukemia 11A) also functions mainly as a transcriptional repressor [55] and down-regulation of $B C L 11 \mathrm{~A}$ has been found mediating the resistance to radiotherapy in the treatment of CRC [56]. HSP90AA1 is closely related to the folding and assembly of many signaling molecules in the Ras/Raf/ERK/1/2 signaling pathway [57]. The binding and dissociation of Hsp90 from these molecules mediate the transformation of these molecules between inactive and active forms [57]. Thus, upregulation of HSP90AA1 after sporamin intervention may suppress the activities of the kinases that drive cancer cell growth and protein synthesis by keeping them in inactive forms. Analysis of the GeneCards ${ }^{\circledR}$ database revealed that NEMF (nuclear export mediator factor) is a component of the ribosome quality control complex and a similar protein in the fly functions as a tumor suppressor but its role in $\mathrm{CRC}$ remains to be explored.

Collectively, it appears that the main changes in the transcriptome of the tumor tissues after sporamin intervention are closely associated with the protein processing in the endoplasmic reticulum. Upregulation of the expression of the molecular chaperones (e.g. HSPH1, HSPA4L) and their cochaperones (e.g. CHORDC1) after sporamin intervention may result from the stresses to cancer cells posed by sporamin intervention. Depending on the specific cellular context, these chaperone proteins may behave both as an oncosuppressor and as a proto-oncogene as is shown in the case of CHORDC1 [58, 59]. However, the current work cannot answer the question of whether these hypotheses are true or not, and more experiments are warranted to investigate the intracellular signal transduction mechanisms involved in cancer cells after sporamin intervention. In addition, regarding the role played by the gut microbiome and its metabolome in the alterations in the transcriptome of the tumor tissues, it is obvious that the current work cannot directly answer this question too and more works are needed in the future to establish a much more solid link between them although this study has provided several clues. Of course, it should also be noted that the gut microbiome may only partly mediates the effect of sporamin because it can also be digested into small peptides, amino acids, and amines, etc. by digestive enzymes and the bacteria in the large intestine. And, once absorbed, their secondary metabolites in the body may also exert functionality at local and/or systemic levels.

\section{Conclusion}

In conclusion, this study is the first that investigated the changes in the gut microbiome and tumor tissue transcriptome after the consumption of sporamin in both healthy and tumor-bearing mice. The findings demonstrated that the consumption of sporamin for four weeks could favorably alter the gut microbiome and its metabolome, improving the gut microenvironment and the viability of the gut microbiota and increasing the detoxification and bioactive substance production activities in the large intestine, by which the host's metabolome may be altered and in turn exerts a suppressing effect on the protein synthesis and growth of tumor tissues. However, more experiments are needed to substantiate these hypotheses in the future.

\section{Abbreviations}


CRC: Colorectal cancer

i.p: intraperitoneally

RNA-seq: RNA sequencing

DMEM: Dulbecco's Modified Eagle's Medium

PCR: polymerase chain reaction

OTUs: operational taxonomic units

PCoA: principal coordinates analysis

PICRUSt: Phylogenetic Investigation of Communities by Reconstruction of Unobserved States

KEGG: Kyoto Encyclopedia of Genes and Genomes

DEGs: Differentially expressed genes

GO: Gene ontology

TF: Transcription factor

PPI: protein-protein interaction

RT-qPCR: Real-time quantitative reverse transcription PCR

SEM: standard error of the mean

GPI: glycosylphosphatidylinositol

SCFA: short-chain fatty acid

ZBED6: zinc finger, BED-type containing 6

BCL11A: B-cell lymphoma/leukemia 11A

NEMF: nuclear export mediator factor

\section{Declarations}

\section{Ethics approval and consent to participate}

Not applicable.

Consent for publication 
Not applicable.

\section{Availability of data and materials}

The datasets used and analyzed during the current study are available from the corresponding author on reasonable request.

\section{Competing interests}

The authors declare that they have no competing interests.

\section{Funding}

This work was supported by the grants from the National Natural Science Foundation of China (No. 81573128 \& 81703216).

\section{Authors' contributions}

PGL conceived and designed the study; GG provided assistance for specimen collection and the guidance of this study; SJC, XPZ and JJZ performed the experiment; CY analyzed data and wrote the manuscript; KWZ and BWC revised the manuscript; PGL had primary responsibility for final content. All authors read and approved the final manuscript.

\section{Acknowledgements}

Authors would like to thank all the teachers in the Department of Nutrition and Food Hygiene, School of Public Health, Capital Medical University, for their help in this study.

\section{References}

1. Bray F, Ferlay J, Soerjomataram I, Siegel RL, Torre LA, Jemal A. Global cancer statistics 2018: GLOBOCAN estimates of incidence and mortality worldwide for 36 cancers in 185 countries. CA Cancer J Clin 2018;68:394-424.

2. Aran V, Victorino AP, Thuler LC, Ferreira CG. Colorectal Cancer: Epidemiology, Disease Mechanisms and Interventions to Reduce Onset and Mortality. Clin Colorectal Cancer 2016;15:195-203.

3. Zou S, Fang L, Lee MH. Dysbiosis of gut microbiota in promoting the development of colorectal cancer. Gastroenterol Rep (Oxf) 2018;6:1-12.

4. Sears CL, Garrett WS. Microbes, microbiota, and colon cancer. CELL HOST MICROBE 2014;15:31728.

5. Kovacs T, Miko E, Ujlaki G, Sari Z, Bai P. The Microbiome as a Component of the Tumor Microenvironment. ADV EXP MED BIOL 2020;1225:137-53. 
6. Fan Y, Pedersen O. Gut microbiota in human metabolic health and disease. NAT REV MICROBIOL 2021;19:55-71.

7. Sarhadi V, Lahti L, Saberi F, Youssef O, Kokkola A, Karla T, Tikkanen M, Rautelin H, Puolakkainen P, Salehi R, Knuutila S. Gut Microbiota and Host Gene Mutations in Colorectal Cancer Patients and Controls of Iranian and Finnish Origin. ANTICANCER RES 2020;40:1325-34.

8. Vetizou M, Pitt JM, Daillere R, Lepage P, Waldschmitt N, Flament C, Rusakiewicz S, Routy B, Roberti MP, Duong CP, Poirier-Colame V, Roux A, Becharef S, Formenti S, Golden E, Cording S, Eberl G, Schlitzer A, Ginhoux F, Mani S, Yamazaki T, Jacquelot N, Enot DP, Berard M, Nigou J, Opolon P, Eggermont A, Woerther PL, Chachaty E, Chaput N, Robert C, Mateus C, Kroemer G, Raoult D, Boneca IG, Carbonnel F, Chamaillard $\mathrm{M}$, Zitvogel L. Anticancer immunotherapy by CTLA-4 blockade relies on the gut microbiota. SCIENCE 2015;350:1079-84.

9. Alexander JL, Wilson ID, Teare J, Marchesi JR, Nicholson JK, Kinross JM. Gut microbiota modulation of chemotherapy efficacy and toxicity. Nat Rev Gastroenterol Hepatol 2017;14:356-65.

10. Amitay EL, Carr PR, Gies A, Laetsch DC, Brenner H. Probiotic/Synbiotic Treatment and Postoperative Complications in Colorectal Cancer Patients: Systematic Review and Meta-analysis of Randomized Controlled Trials. Clin Transl Gastroenterol 2020;11:e268.

11. Gong Y, Dong R, Gao X, Li J, Jiang L, Zheng J, Cui S, Ying M, Yang B, Cao J, He Q. Neohesperidin prevents colorectal tumorigenesis by altering the gut microbiota. PHARMACOL RES 2019;148:104460.

12. Sanders ME, Merenstein DJ, Reid G, Gibson GR, Rastall RA. Probiotics and prebiotics in intestinal health and disease: from biology to the clinic. Nat Rev Gastroenterol Hepatol 2019;16:605-16.

13. Gibson GR, Hutkins R, Sanders ME, Prescott SL, Reimer RA, Salminen SJ, Scott K, Stanton C, Swanson KS, Cani PD, Verbeke K, Reid G. Expert consensus document: The International Scientific Association for Probiotics and Prebiotics (ISAPP) consensus statement on the definition and scope of prebiotics. Nat Rev Gastroenterol Hepatol 2017;14:491-502.

14. Hattori T, Nakagawa T, Maeshima M, Nakamura K, Asahi T. Molecular cloning and nucleotide sequence of CDNA for sporamin, the major soluble protein of sweet potato tuberous roots. PLANT MOL BIOL 1985;5:313-20.

15. Qian CJ, Qi YX, Chen XY, Zeng JP, Yao J. Sporamin suppresses growth of human esophageal squamous cell carcinoma cells by inhibition of NFkappaB via an AKTindependent pathway. MOL MED REP 2017;16:9620-26.

16. Qian C, Chen X, Qi Y, Zhong S, Gao X, Zheng W, Mao Z, Yao J. Sporamin induces apoptosis and inhibits NF-kappaB activation in human pancreatic cancer cells. Tumour Biol 2017;39:1393382747. 
17. Yao J, Qian C. Sporamin induce apoptosis in human tongue carcinoma cells by down-regulating Akt/GSK-3 signaling. Fundam Clin Pharmacol 2011;25:229-36.

18. Yang C, Zhang JJ, Zhang XP, Xiao R, Li PG. Sporamin suppresses growth of xenografted colorectal carcinoma in athymic BALB/c mice by inhibiting liver beta-catenin and vascular endothelial growth factor expression. World J Gastroenterol 2019;25:3196-206.

19. Qian CJ, Qi YX, Zhong S, Zeng JP, Chen XY, Yao J. Mitogen-activated protein kinase inhibition enhances the antitumor effects of sporamin in human pancreatic cancer cells. ONCOL LETT 2018;16:1237-42.

20. Zhi-dong, XIONG, And, Peng-gao, LI, And, Tai-hua, MU. The Differentiation- and ProliferationInhibitory Effects of Sporamin from Sweet Potato in 3T3-L1 Preadipocytes. Agricultural Sciences in China 2009.

21. Caporaso JG, Kuczynski J, Stombaugh J, Bittinger K, Bushman FD, Costello EK, Fierer N, Pena AG, Goodrich JK, Gordon JI, Huttley GA, Kelley ST, Knights D, Koenig JE, Ley RE, Lozupone CA, McDonald D, Muegge BD, Pirrung M, Reeder J, Sevinsky JR, Turnbaugh PJ, Walters WA, Widmann J, Yatsunenko T, Zaneveld J, Knight R. QIIME allows analysis of high-throughput community sequencing data. NAT METHODS 2010;7:335-36.

22. Edgar RC, Haas BJ, Clemente JC, Quince C, Knight R. UCHIME improves sensitivity and speed of chimera detection. BIOINFORMATICS 2011;27:2194-200.

23. Edgar RC. UPARSE: highly accurate OTU sequences from microbial amplicon reads. NAT METHODS 2013;10:996-98.

24. Cole JR, Wang Q, Fish JA, Chai B, McGarrell DM, Sun Y, Brown CT, Porras-Alfaro A, Kuske CR, Tiedje JM. Ribosomal Database Project: data and tools for high throughput rRNA analysis. NUCLEIC ACIDS RES 2014;42:D633-42.

25. Westcott SL, Schloss PD. De novo clustering methods outperform reference-based methods for assigning 16S rRNA gene sequences to operational taxonomic units. PEERJ 2015;3:e1487.

26. Langille MG, Zaneveld J, Caporaso JG, McDonald D, Knights D, Reyes JA, Clemente JC, Burkepile DE, Vega TR, Knight R, Beiko RG, Huttenhower C. Predictive functional profiling of microbial communities using 16S rRNA marker gene sequences. NAT BIOTECHNOL 2013;31:814-21.

27. Parks DH, Tyson GW, Hugenholtz P, Beiko RG. STAMP: statistical analysis of taxonomic and functional profiles. BIOINFORMATICS 2014;30:3123-24.

28. Cock PJA, Fields CJ, Goto N, Heuer ML, Rice PM. The Sanger FASTQ file format for sequences with quality scores, and the Solexa/Illumina FASTQ variants. NUCLEIC ACIDS RES 2010;38:1767-71. 
29. Langmead B, Salzberg SL. Fast gapped-read alignment with Bowtie 2. NAT METHODS 2012;9:357-

59.

30. Li B, Dewey CN. RSEM: accurate transcript quantification from RNA-Seq data with or without a reference genome. BMC BIOINFORMATICS 2011;12:323.

31. Guda MR, Velpula KK, Asuthkar S, Cain CP, Tsung AJ. Targeting RGS4 Ablates Glioblastoma Proliferation. INT J MOL SCI 2020;21.

32. Buchfink B, Xie C, Huson DH. Fast and sensitive protein alignment using DIAMOND. NAT METHODS 2015;12:59-60.

33. von Mering C, Jensen LJ, Snel B, Hooper SD, Krupp M, Foglierini M, Jouffre N, Huynen MA, Bork P. STRING: known and predicted protein-protein associations, integrated and transferred across organisms. NUCLEIC ACIDS RES 2005;33:D433-37.

34. Killcoyne S, Carter GW, Smith J, Boyle J. Cytoscape: a community-based framework for network modeling. Methods Mol Biol 2009;563:219-39.

35. Markowitz SD, Bertagnolli MM. Molecular origins of cancer: Molecular basis of colorectal cancer. N Engl J Med 2009;361:2449-60.

36. McKnite AM, Perez-Munoz ME, Lu L, Williams EG, Brewer S, Andreux PA, Bastiaansen JW, Wang X, Kachman SD, Auwerx J, Williams RW, Benson AK, Peterson DA, Ciobanu DC. Murine gut microbiota is defined by host genetics and modulates variation of metabolic traits. PLOS ONE 2012;7:e39191.

37. van den Elsen LW, Poyntz HC, Weyrich LS, Young W, Forbes-Blom EE. Embracing the gut microbiota: the new frontier for inflammatory and infectious diseases. Clin Transl Immunology 2017;6:e125.

38. Zhou Z, Chen J, Yao H, Hu H. Fusobacterium and Colorectal Cancer. FRONT ONCOL 2018;8:371.

39. Grivennikov SI, Wang K, Mucida D, Stewart CA, Schnabl B, Jauch D, Taniguchi K, Yu GY, Osterreicher CH, Hung KE, Datz C, Feng Y, Fearon ER, Oukka M, Tessarollo L, Coppola V, Yarovinsky F, Cheroutre H, Eckmann L, Trinchieri G, Karin M. Adenoma-linked barrier defects and microbial products drive IL-23/IL-17-mediated tumour growth. NATURE 2012;491:254-58.

40. Davani-Davari D, Negahdaripour M, Karimzadeh I, Seifan M, Mohkam M, Masoumi SJ, Berenjian A, Ghasemi Y. Prebiotics: Definition, Types, Sources, Mechanisms, and Clinical Applications. Foods 2019;8.

41. Cardona F, Andres-Lacueva C, Tulipani S, Tinahones FJ, Queipo-Ortuno MI. Benefits of polyphenols on gut microbiota and implications in human health. J NUTR BIOCHEM 2013;24:1415-22. 
42. Touchefeu Y, Duchalais E, Bruley DVS, Alameddine J, Mirallie E, Matysiak-Budnik T, Le Bastard Q, Javaudin F, Rimbert M, Jotereau F, Montassier E. Concomitant decrease of double-positive lymphocyte population CD4CD8alphaalpha and Faecalibacterium prausnitzii in patients with colorectal cancer. Eur J Gastroenterol Hepatol 2021;32:149-56.

43. Hu Y, Le Leu RK, Christophersen CT, Somashekar R, Conlon MA, Meng XQ, Winter JM, Woodman RJ, McKinnon R, Young GP. Manipulation of the gut microbiota using resistant starch is associated with protection against colitis-associated colorectal cancer in rats. CARCINOGENESIS 2016;37:366-75.

44. Ambalam P, Raman M, Purama RK, Doble M. Probiotics, prebiotics and colorectal cancer prevention. Best Pract Res Clin Gastroenterol 2016;30:119-31.

45. Oh NS, Lee JY, Kim YT, Kim SH, Lee JH. Cancer-protective effect of a synbiotic combination between Lactobacillus gasseri 505 and a Cudrania tricuspidata leaf extract on colitis-associated colorectal cancer. Gut Microbes 2020;12:1785803.

46. Luan Y, Li Y, Yue X, Cao Y, Xiang F, Mao D, Xiong Z. Metabonomics of mice intestine in Codonopsis foetens induced apoptosis of intestine cancer cells. SAUDI J BIOL SCI 2019;26:1003-10.

47. Yachida S, Mizutani S, Shiroma H, Shiba S, Nakajima T, Sakamoto T, Watanabe H, Masuda K, Nishimoto Y, Kubo M, Hosoda F, Rokutan H, Matsumoto M, Takamaru H, Yamada M, Matsuda T, Iwasaki M, Yamaji T, Yachida T, Soga T, Kurokawa K, Toyoda A, Ogura Y, Hayashi T, Hatakeyama M, Nakagama H, Saito Y, Fukuda S, Shibata T, Yamada T. Metagenomic and metabolomic analyses reveal distinct stagespecific phenotypes of the gut microbiota in colorectal cancer. NAT MED 2019;25:968-76.

48. Golding BT, Watson WP. Possible mechanisms of carcinogenesis after exposure to benzene. IARC Sci Publ 1999:75-88.

49. Arima K, Lau MC, Zhao M, Haruki K, Kosumi K, Mima K, Gu M, Vayrynen JP, Twombly TS, Baba Y, Fujiyoshi K, Kishikawa J, Guo C, Baba H, Richards WG, Chan AT, Nishihara R, Meyerhardt JA, Nowak JA, Giannakis M, Fuchs CS, Ogino S. Metabolic Profiling of Formalin-Fixed Paraffin-Embedded Tissues Discriminates Normal Colon from Colorectal Cancer. MOL CANCER RES 2020.

50. Castellaro AM, Tonda A, Cejas HH, Ferreyra H, Caputto BL, Pucci OA, Gil GA. Oxalate induces breast cancer. BMC CANCER 2015;15.

51. Shiby VK, Mishra HN. Fermented milks and milk products as functional foods-a review. Crit Rev Food Sci Nutr 2013;53:482-96.

52. Franz CMAP, Holzapfel WH, Stiles ME. Enterococci at the crossroads of food safety?. Netherlands: Elsevier B.V; 1999: 1-24.

53. Metabolic inhibitors of bacterial glycan biosynthesis. CHEM SCI 2020. 
54. Akhtar AM, Younis S, Wallerman O, Gupta R, Andersson L, Sjoblom T. Transcriptional modulator ZBED6 affects cell cycle and growth of human colorectal cancer cells. Proc Natl Acad Sci U S A 2015;112:7743-48.

55. Lazarus KA, Hadi F, Zambon E, Bach K, Santolla MF, Watson JK, Correia LL, Das M, Ugur R, Pensa S, Becker L, Campos LS, Ladds G, Liu P, Evan GI, McCaughan FM, Le Quesne J, Lee JH, Calado D, Khaled WT. BCL11A interacts with SOX2 to control the expression of epigenetic regulators in lung squamous carcinoma. NAT COMMUN 2018;9:3327.

56. Park SY, Lee SJ, Cho HJ, Kim JT, Yoon HR, Lee KH, Kim BY, Lee Y, Lee HG. Epsilon-Globin HBE1 Enhances Radiotherapy Resistance by Down-Regulating BCL11A in Colorectal Cancer Cells. Cancers (Basel) 2019;11.

57. Zuehlke AD, Beebe K, Neckers L, Prince T. Regulation and function of the human HSP90AA1 gene. GENE 2015;570:8-16.

58. Brancaccio M, Rocca S, Secli L, Busso E, Fusella F. The double face of Morgana in tumorigenesis. Oncotarget 2015;6:42603-12.

59. Di Savino A, Panuzzo C, Rocca S, Familiari U, Piazza R, Crivellaro S, Carra G, Ferretti R, Fusella F, Giugliano E, Camporeale A, Franco I, Miniscalco B, Cutrin JC, Turco E, Silengo L, Hirsch E, Rege-Cambrin G, Gambacorti-Passerini C, Pandolfi PP, Papotti M, Saglio G, Tarone G, Morotti A, Brancaccio M. Morgana acts as an oncosuppressor in chronic myeloid leukemia. BLOOD 2015;125:2245-53.

\section{Figures}


TCG
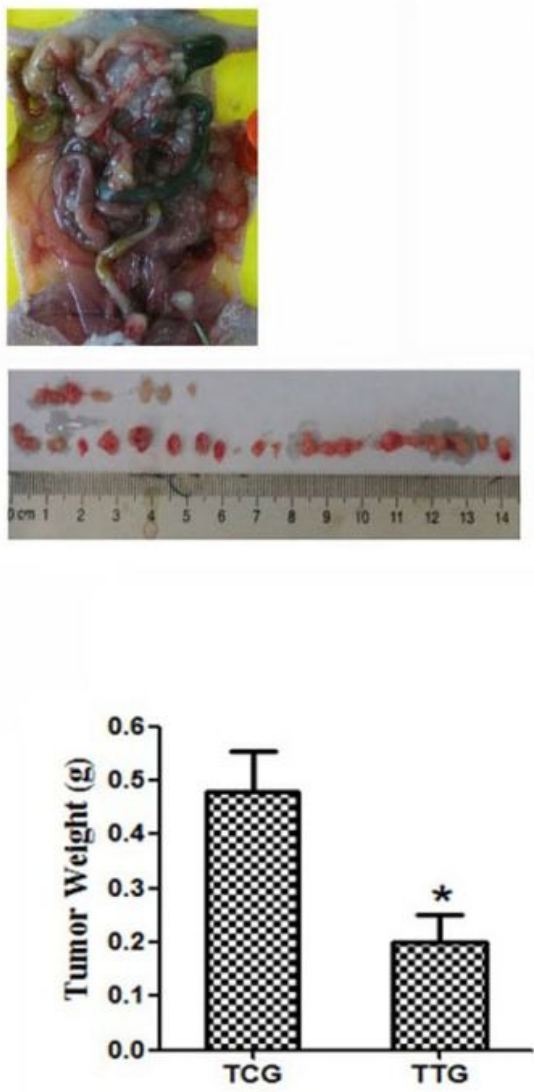

TTG
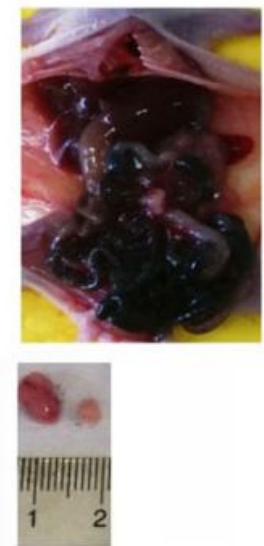

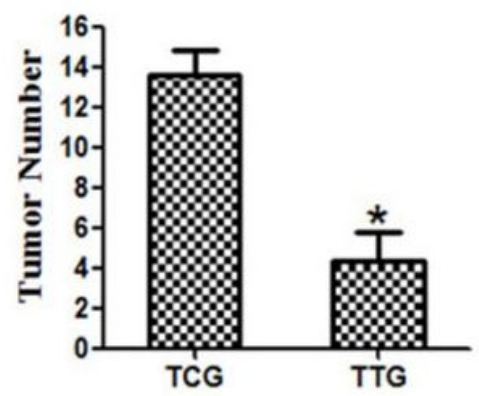

Figure 1

The body weight (BW) and tumor burden of the mice after 28 days of treatment with or without sporamin. (A) BW of the healthy (CG1 and CG2) and tumor-bearing (TCG and TTG) mice during the experiment period. (B) Representative images of the abdominal tumors of tumor-bearing mice after 28 days of treatment with either vehicle (TCG) or sporamin (TTG) with separate images (lower panel) showing their multiplicity and sizes. (C) Comparison of the tumor number and weight between the TCG and TTG group. Data shown are mean \pm SEM $(n=3)$. CG1: healthy mice treated with vehicle (control); CG2: healthy mice treated with sporamin; TCG: tumor-bearing mice treated with vehicle; TTG: tumor-bearing mice treated with sporamin. 
A

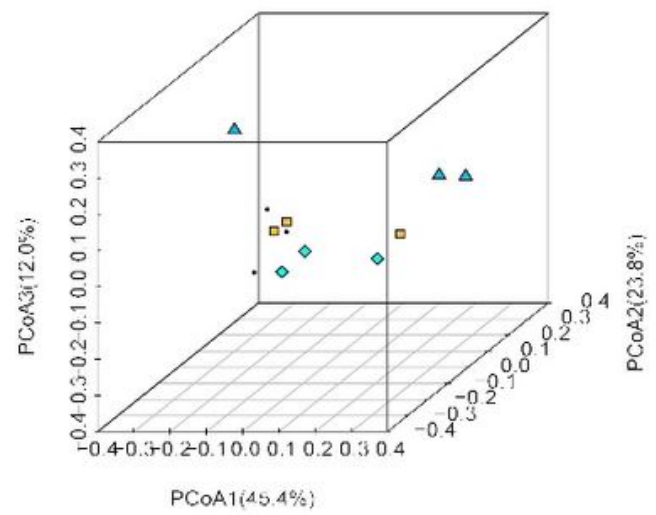

C

\section{heatmap of phylum}

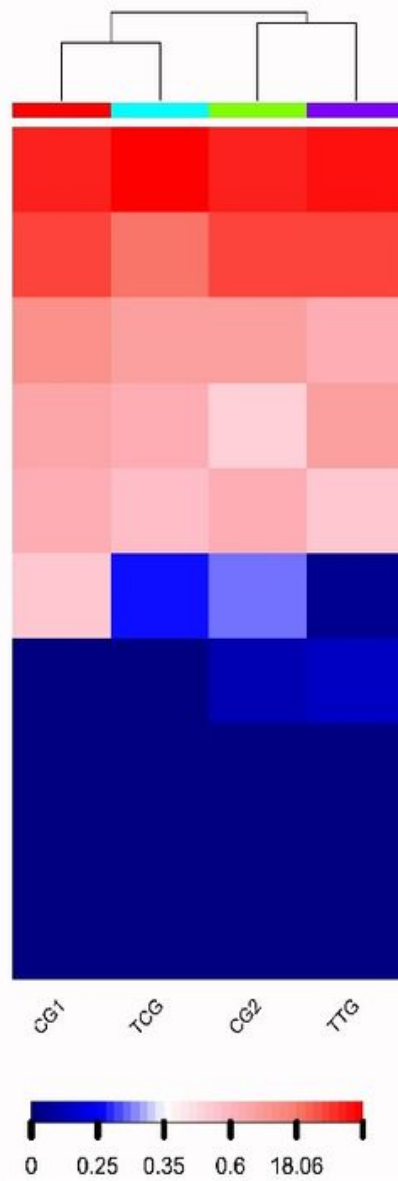

B

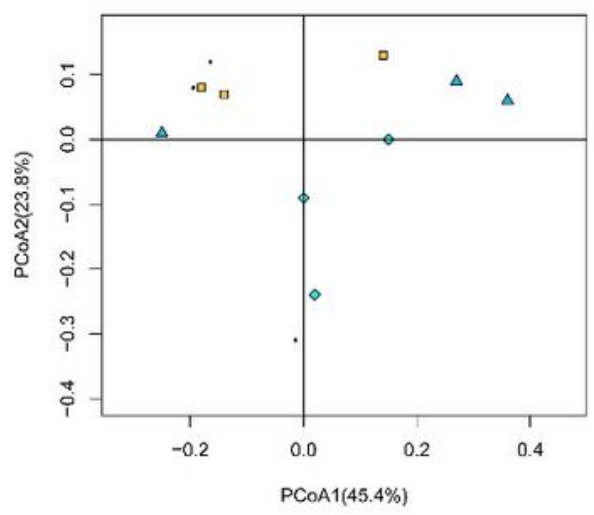

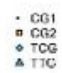

heatmap of genus

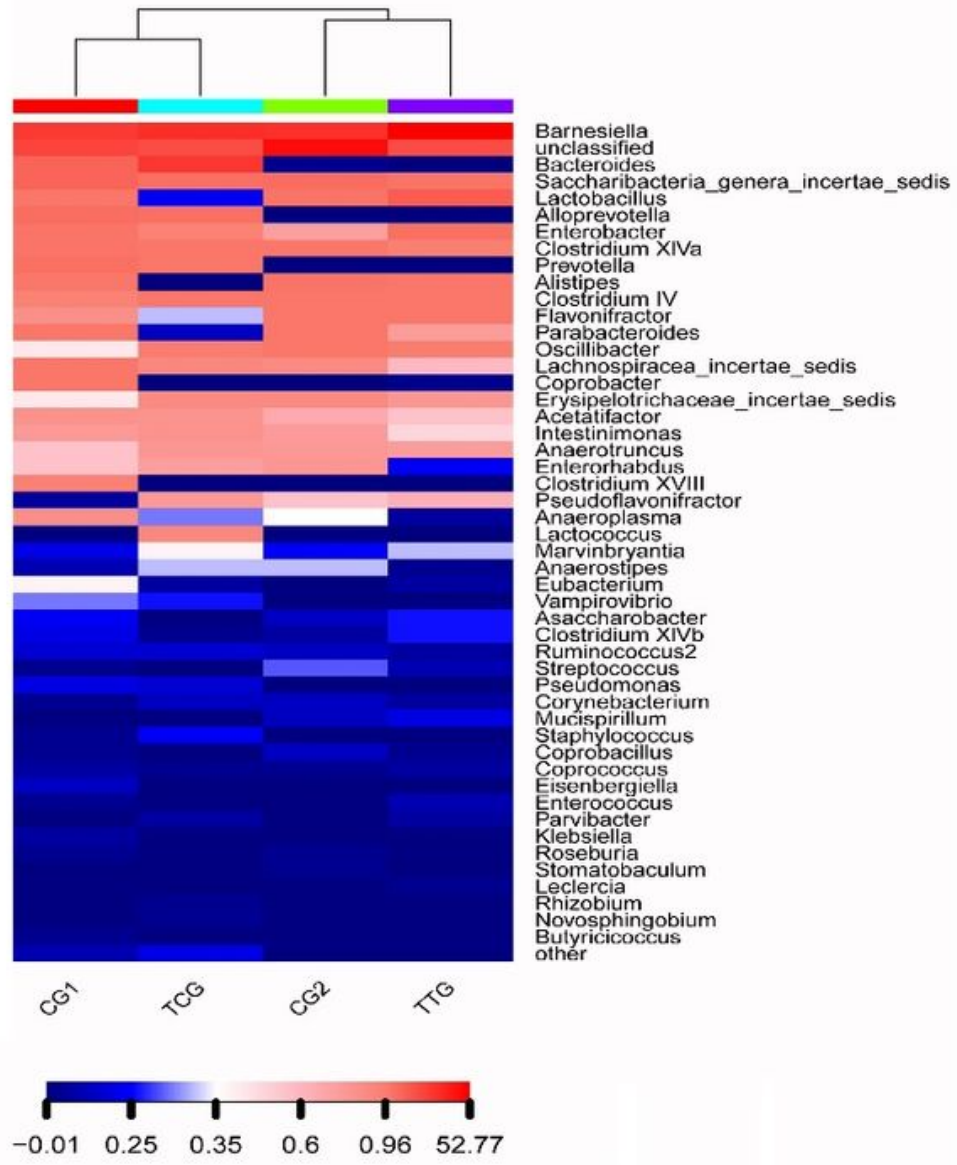

\section{Figure 2}

Sporamin changed the beta-diversity of the fecal microbiota in tumor-bearing (TCG and TTG) and healthy (CG1 and CG2) mice. Mice were treated with (CG1 and TTG) or without (CG1 and TCG) sporamin for 28 days. (A) Three-dimensional principal coordinate analysis (PCOA) plot showing the overall dissimilarities in the fecal microbiota structures across the four groups. (B) Two-dimensional PCoA plot showing the separation of the fecal samples from the four groups. (C) Hierarchically clustered heat maps showing the 
distribution of bacterial phyla (left panel) and genera (right panel) across the four groups. Clusters based on the taxonomic distances of samples are indicated along the X-axis on the top of the heatmap. In the color legends at the bottom of the plots, the redder the color the higher the relative abundance, the bluer the color the lower the relative abundance. CG1: healthy mice treated with vehicle (control); CG2: healthy mice treated with sporamin; TCG: tumor-bearing mice treated with vehicle; TTG: tumor-bearing mice treated with sporamin.

A
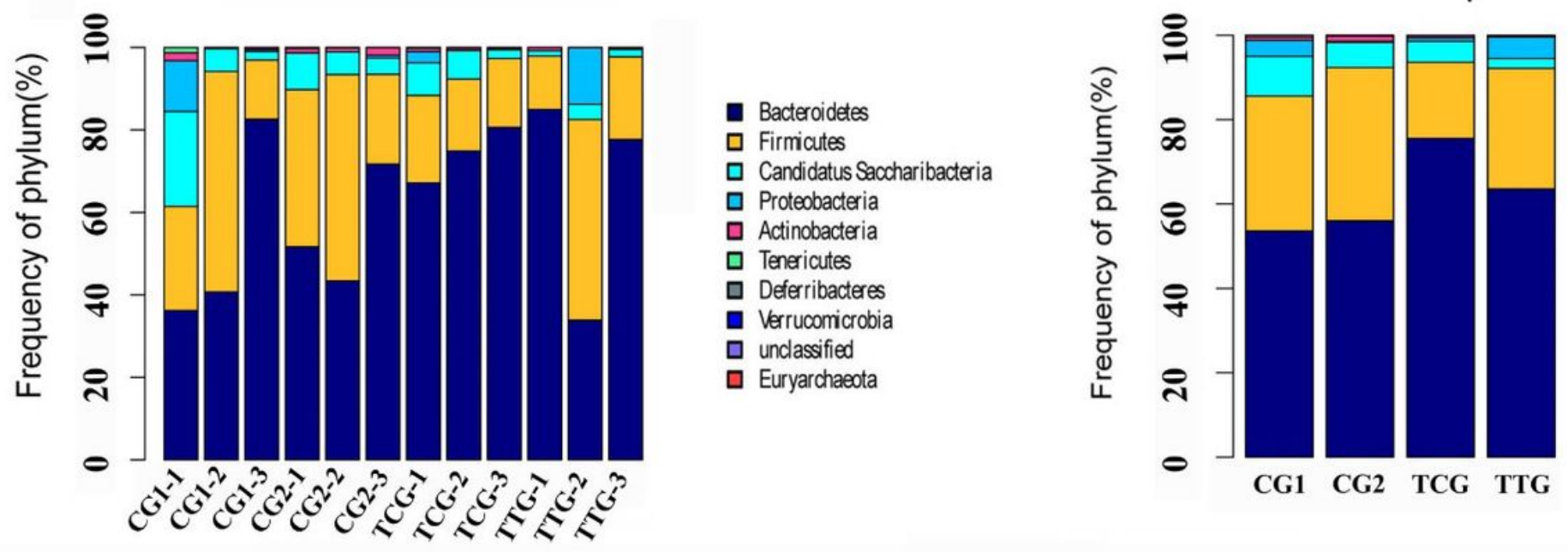

B
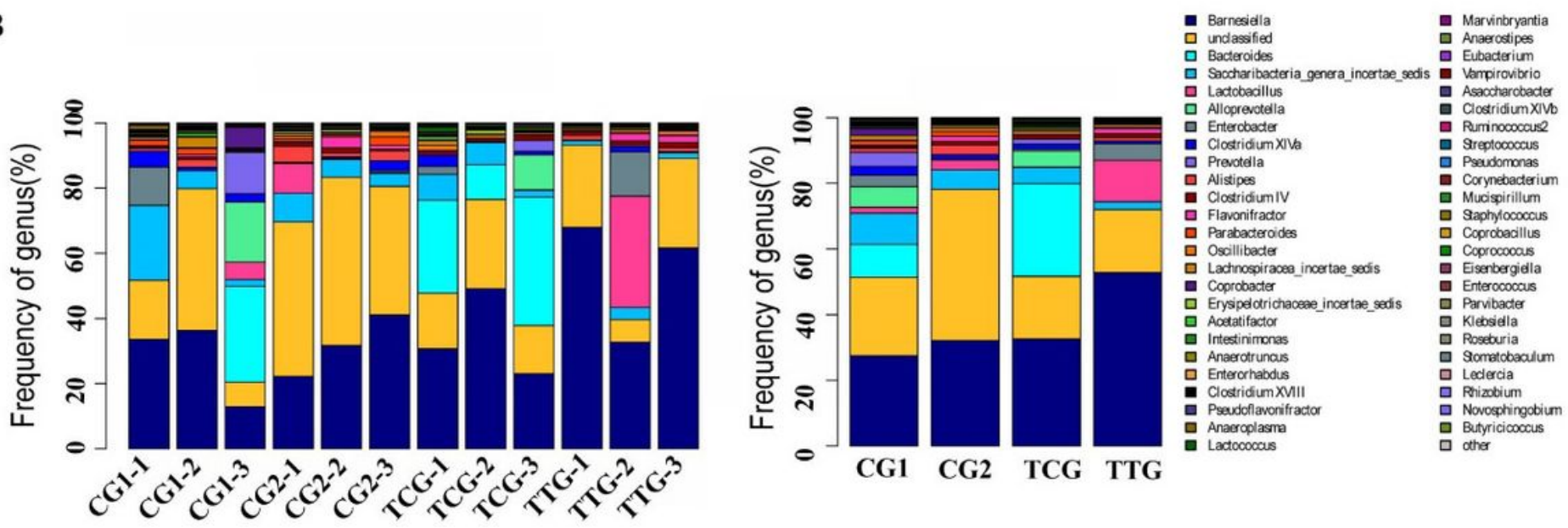

Figure 3

Distribution histograms showing the compositions of the fecal microbiota at the phylum and genus levels. (A) shows the phylum level while (B) shows the genus level. The left panels represent the bacterial taxonomic composition for individual samples where the number in the sample name represents the code for each sample. The right panels represent the bacterial taxonomic compositions for the four groups. CG1: healthy mice treated with vehicle (control); CG2: healthy mice treated with sporamin; TCG: tumorbearing mice treated with vehicle; TTG: tumor-bearing mice treated with sporamin. 
A

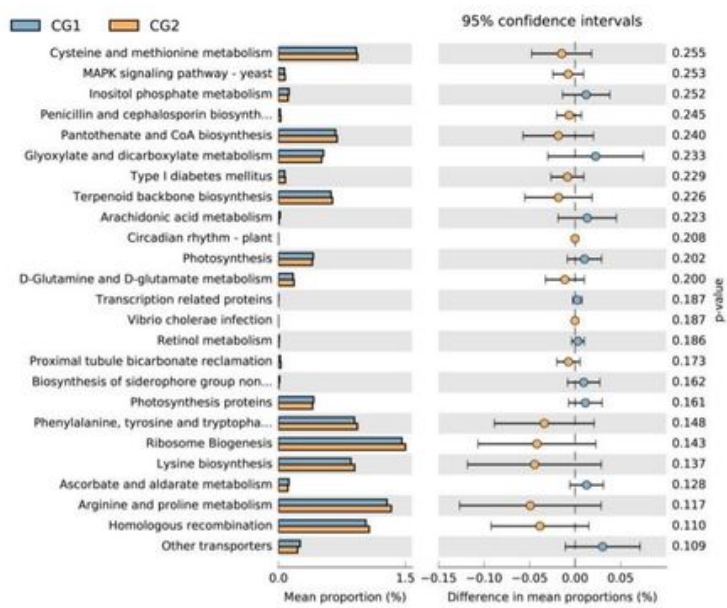

B

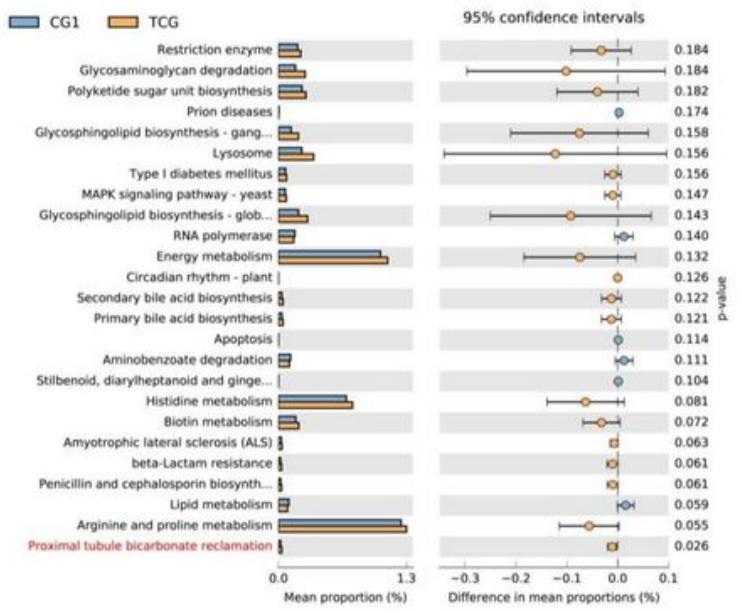

C

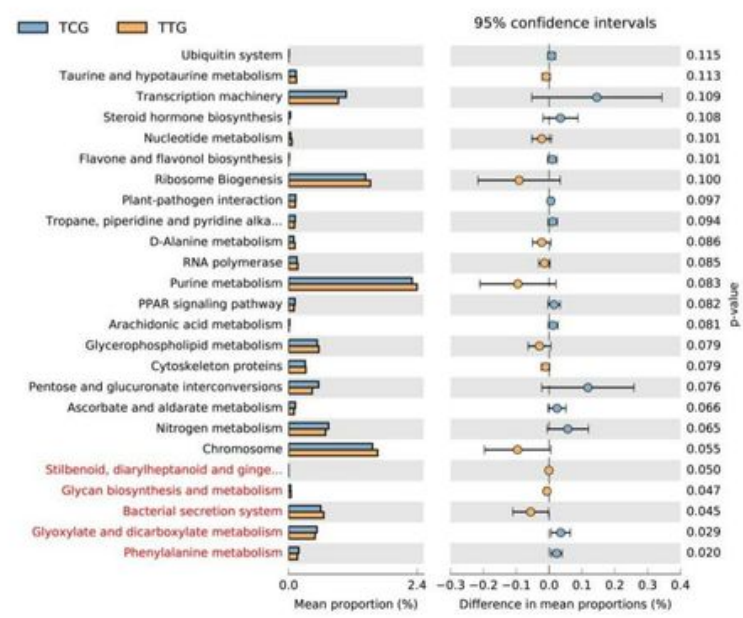

\section{Figure 4}

Top 25 predicted metabolic pathways that were influenced by the gut microbiome in mice exposed to different experimental treatments. (A) CG1 vs. CG2; (B) CG1 vs. TCG; (C) TCG vs. TTG. The left abscissa shows the mean proportion of the enriched function. The right abscissa shows the difference in the mean proportion of the functional pathways between the two groups and their $95 \%$ confidence interval. The ordinate on the right is the P-value obtained by comparing the two groups. The pathway names on the 
left ordinate are arranged in ascending order of P-values from bottom to top. The pathways with P-values lower than 0.05 are displayed in red. CG1: healthy mice treated with vehicle (control); CG2: healthy mice treated with sporamin; TCG: tumor-bearing mice treated with vehicle; TTG: tumor-bearing mice treated with sporamin.

A

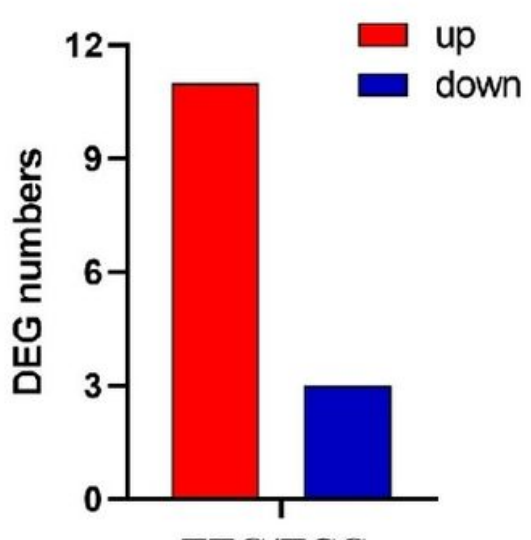

TTG/TCG

C
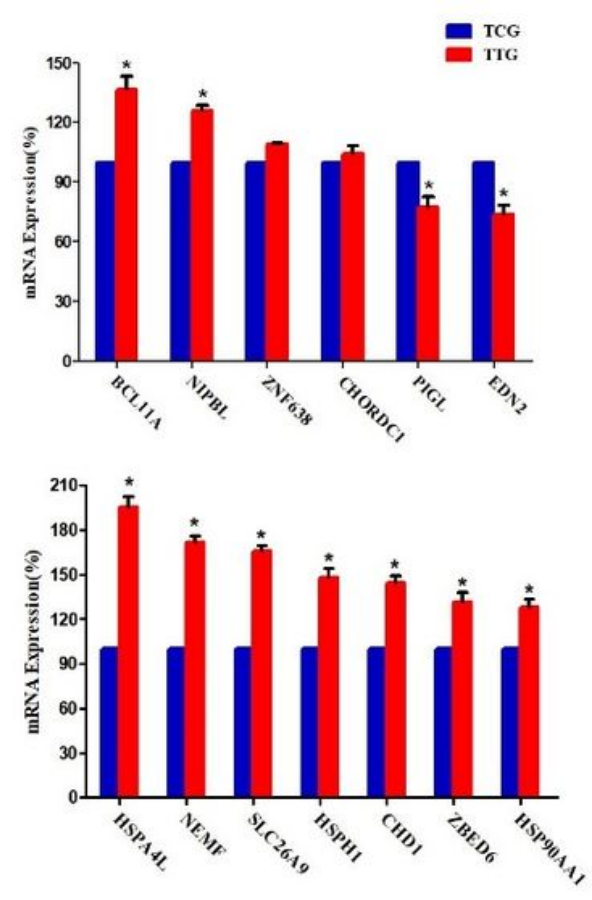

\section{B}

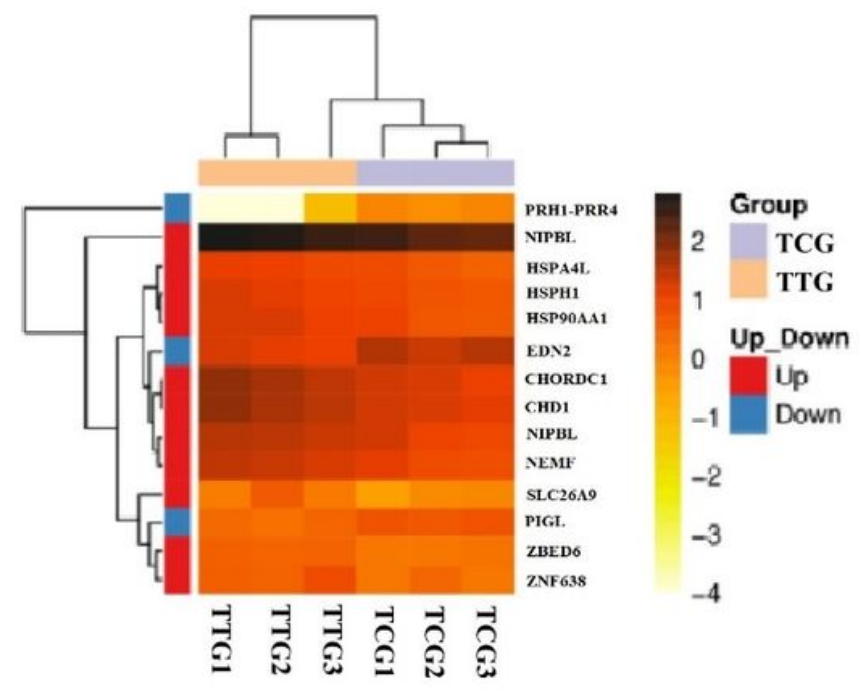

D

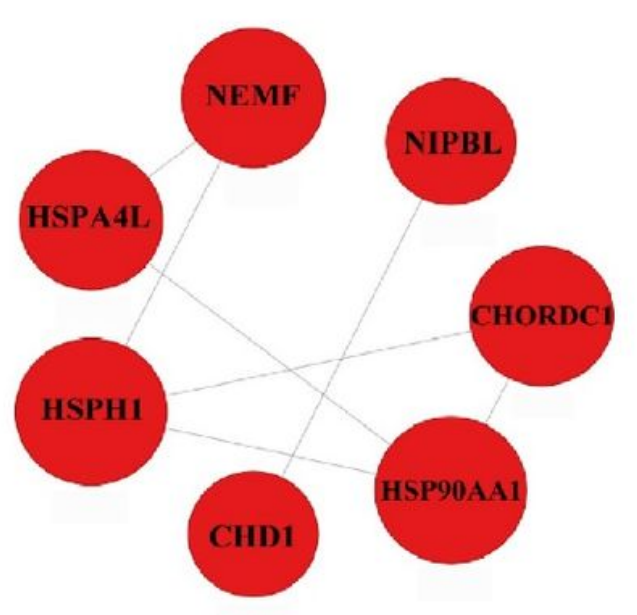

\section{Figure 5}

The differentially expressed genes (DEGs) after 28 days of sporamin intervention in tumor-bearing mice and their interactions. (A) Summary of the numbers of the up- and down-regulated DEGs between the TTG and TCG groups. (B) Hierarchically clustered heatmap showing the expression patterns of the DEGs between TTG and TCG. The X-axis represents the individual samples and their clusters. Y-axis represents 
the up- (red) and down-regulated (blue) DEGs and their clusters. The color represents the log10 transformed gene expression levels. The darker the color the higher the expression level while the lighter the color the lower the expression level. (C) RT-qPCR validation of the changes in the expression of the DEGs between TTG and TCG. (D) The protein-protein interaction (PPI) network is predicted based on the DEGs between the TTG and TCG groups. The red dots refer to up-regulated DEG. Their interactions are shown by the edges linking them together. The size of the circles indicates the number of interactions with that gene. ${ }^{*} P<0.05$ vs. TCG. TCG: tumor-bearing mice treated with vehicle; TTG: tumor-bearing mice treated with sporamin.
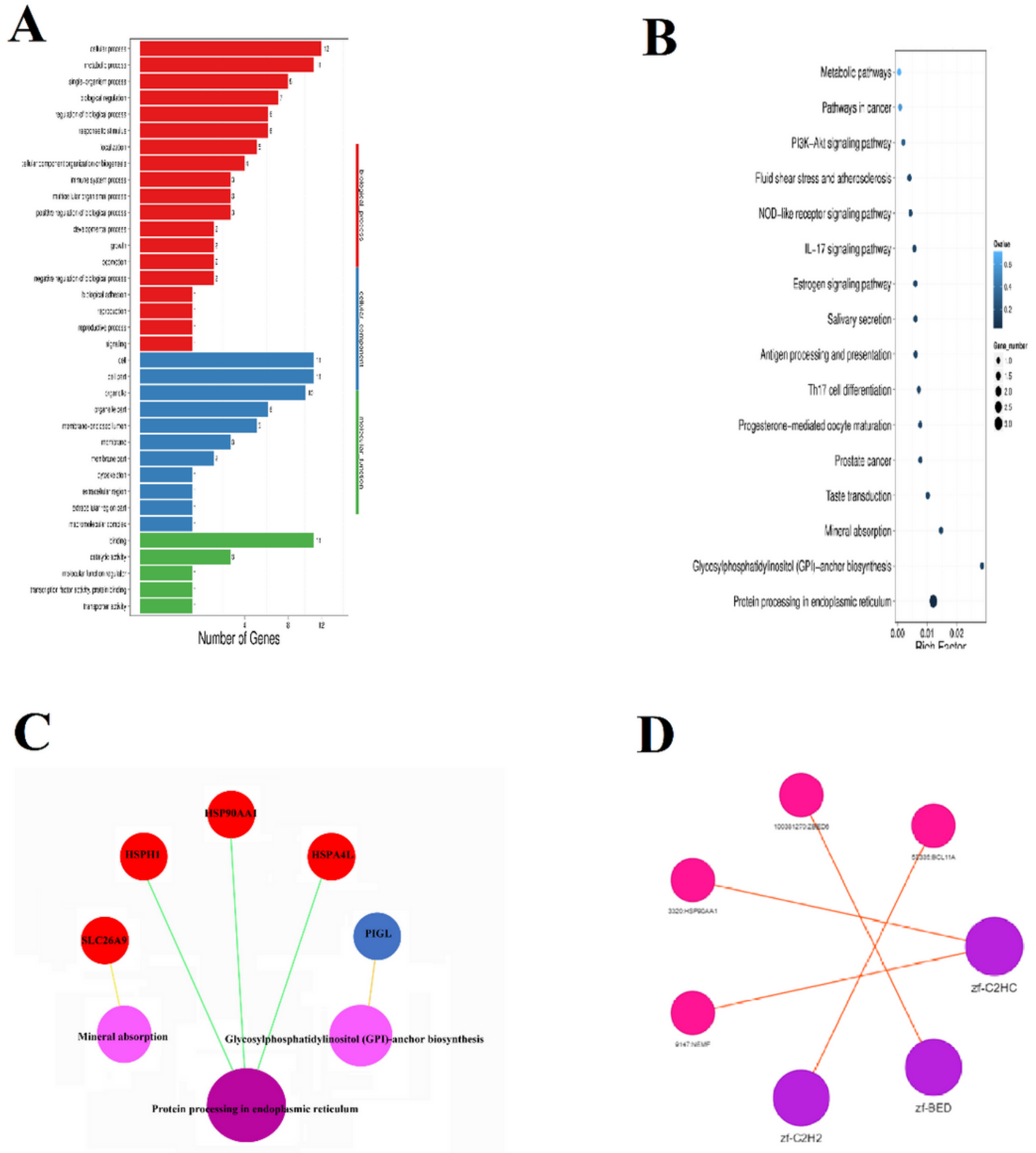


\section{Figure 6}

The Gene Ontology (GO) analysis and the pathway enrichment analysis based on the KEGG database according to the DEGs between the TTG and TCG groups. (A) The significantly enriched GO terms, which are classified as the cellular component (CC), biological processes (BP), and molecular functions (MF), are annotated according to the DEGs between the TTG and TCG groups. The X-axis represents the number of DEGs. The Y-axis represents the GO terms. (B) The KEGG pathway enrichment analysis basing on the DEGs. The X-axis represents the Rich Factor that is the quotient of foreground value (the number of DEGs) and background value (total gene number); the larger the value the more significant enrichment. The $\mathrm{Y}$-axis represents the enriched pathway names. The blue color of the dots indicates the q-value; the darker the color the lower the q-value and the more significant the enrichment. The size of the dots represents the number of the genes enriched in that pathway; the bigger the size the larger the number. (C) The KEGG-DEG relationship network analysis. The red and blue dots on the upper panel represent the up- and down-regulated DEGs, respectively. The purple dots on the lower panel indicate the enriched KEGG pathways. Their relationships with the DEGs are shown by the lines that link them together; the larger the dot-area the higher the degree of enrichment. (D) The transcription factor (TF) prediction basing on the DEGs between the TTG and the TCG group. The pink dots represent the up-regulated DEGs. The purple dots represent the predicted TF domains; the larger the size of the dots, the more DEGs the TF modulates.

\section{Supplementary Files}

This is a list of supplementary files associated with this preprint. Click to download.

- floatimage7.jpeg 